\title{
PROTO-PLANETARY NEBULAE
}

\author{
BRUCE J. HRIVNAK \\ Valparaiso University \\ Valparaiso, IN 46383, USA
}

\begin{abstract}
.
We review the properties of proto-planetary nebulae, highlighting the advances in our quantitative knowledge of these objects. The discussion focuses on the chemistry and morphology of their circumstellar envelopes and the chemistry and variability of their central stars.
\end{abstract}

\section{Introduction}

In this presentation, we will review our current knowledge of proto-planetary nebulae (PPNe), objects in transition between the asymptotic giant branch (AGB) and planetary nebula (PN) phases. A PPN consists of a central star evolving to the left on the HR diagram, surrounded by a detached, expanding circumstellar envelope (CSE) of gas and dust. Observations reveal a "double-peaked" spectral energy distribution (SED), composed of light from a reddened photosphere and re-emission from circumstellar dust (see Hrivnak, Kwok, \& Volk 1989). There has been much research in this field since the last PN conference (Kwok 1993a,b), and we will attempt to highlight this. Some has continued to be of a quantitative nature, with the identification and classification of new PPNe, but an increasing amount has been of a quantitative nature, with regard to both the CSE and the central star.

Observationally, the coolest PPN stars appear to have a spectral type of late-G, or a temperature $\sim 5000 \mathrm{~K}$ (Hrivnak 1995). Photoionization occurs when the temperature of the central star reaches $\sim 30,000 \mathrm{~K}$, and we will regard this as the beginning of the $\mathrm{PN}$ stage. This sets the approximate temperature and spectral ranges expected for PPNe. Theoretical studies show that the transition times scale for the PPN phase, from the time when extreme mass loss on the AGB has ended (and the remaining envelope 


\section{B. Hrivnak}

mass is $<10^{-3} \mathrm{M}_{\odot}$ ) and photoionization begins, is small, $\sim 10^{3} \mathrm{yrs}$ (Blöcker 1995).

\section{Circumstellar Envelopes: Chemistry}

\subsection{MOLECULAR-LINE STUDIES}

PPN candidates have continued to be observed in $\mathrm{OH}, \mathrm{CO}$, and HCN. From these, Omont et al. (1993) determined criteria to allow one to classify the chemistry $(\mathrm{C}$ or $\mathrm{O})$ of the objects on the basis of the ratio of $\mathrm{CO} / \mathrm{HCN}$ : for $\mathrm{C}$-rich sources, $\mathrm{CO}(1-0) / \mathrm{HCN}<5$ or $\mathrm{CO}(2-1) / \mathrm{HCN}<12$. Additional molecules have also been detected.

\subsection{VISIBLE SPECTROSCOPY}

Hrivnak (1995) identified molecular $\mathrm{C}_{2}$ and $\mathrm{C}_{3}$ absorption features in 8 PPN candidates, in addition to the Egg nebula, a previously known source. These are found in $\mathrm{G}$ and even some $\mathrm{F}$ stars, where the temperature is too high for them to be photospheric.

With observations at higher resolution, Bakker et al. $(1996,1997$; also Reddy et al. 1996) have made a detailed study of molecular absorption features in all 9 of these $\mathrm{PPNe}$, plus some additional post-AGB stars. They identified the vibrational bands of $\mathrm{C}_{2}$ and $\mathrm{CN}$ in all nine. (These will be listed later in Table 1). From these, they were able to determine expansion velocities for the molecules of $5-44 \mathrm{~km} \mathrm{~s}^{-1}$, values which agree very well with the $\mathrm{CO}$ millimeter-line expansion velocities, and rotational temperatures of $43-399 \mathrm{~K}$ for $\mathrm{C}_{2}$ and $18-50 \mathrm{~K}$ for $\mathrm{CN}$. This clearly identifies the $\mathrm{CSE}$ as the location of these molecules. Column densities for the molecules were determined and mass loss rates estimated. Thus the application of high-resolution optical spectroscopy provides a new and useful probe of the chemical and physical properties of the CSEs in PPNe, at least in C-rich ones.

\subsection{NEAR-INFRARED SPECTROSCOPY}

Near-infrared spectra have been obtained of a number of PPNe (Hrivnak, Kwok, \& Geballe 1994; Oudmaijer et al. 1995). A few are found to show $\mathrm{CO}$ in emission at $2.3 \mu \mathrm{m}$, and for IRAS $22272+5435$, the CO varied from emission to absorption over a 3 month period. Emission at 3.3 and $3.4 \mu \mathrm{m}$, commonly attributed to polycyclic aromatic hydrocarbons (PAHs), is seen in several carbon-rich PPNe. In a few of these, the $3.4 \mu \mathrm{m}$ feature is unusually strong compared with the usually dominant $3.3 \mu \mathrm{m}$ feature (Geballe $e t$ al. 1992). 


\section{PROTO-PLANETARY NEBULAE}

\subsection{MID-INFRARED SPECTROSCOPY}

IRAS: The IRAS LRS spectra provided an opportunity to classify the chemistry of the CSEs of evolved stars on the basis of the presence of silicate features at 9.8 and $18 \mu \mathrm{m}$ or silicon-carbide features at $11.3 \mu \mathrm{m}$. Indeed, the 9.8 and $18 \mu \mathrm{m}$ features have been seen in emission in several PPN candidates. While the silicon-carbide feature has not been seen, several other features have been identified which are indicative of a C-rich CSE.

$P A H s$ : Strong UIR features at $6.2,7.7,8.6,11.3 \mu \mathrm{m}$, attributed to PAHs, have been seen in several C-rich, typically young PNe. In PPNe, the feature at $7.7 \mu \mathrm{m}$ appears to be at the edge of the IRAS LRS spectra in several PPNe. By using KAO, Buss et al. (1990) identified strong infrared emission features at $6.9,8$, and $12 \mu \mathrm{m}$. In these cool objects, it is suggested that visible photons pump the PAH bands. Recently, using mid-infrared spectra obtained with the UKIRT CGS3 spectrometer, Justtanont et al. (1996) observed several carbon-rich PPNe and found in addition to the standard UIR features new features at $7.9,8.2,10.6,11.5$, and $12.2 \mu \mathrm{m}$, which they attribute to the PAH molecule chrysene.

$21 \mu \mathrm{m}$ : The " $21 \mu \mathrm{m}$ " emission feature was first identified by Kwok, Volk, \& Hrivnak (1989) in the IRAS LRS spectra of 4 PPNe.Associated with it is a flat "plateau" in the spectrum from $12-18 \mu \mathrm{m}$, emission from $11-12 \mu \mathrm{m}$, and perhaps an emission feature at $\sim 8 \mu \mathrm{m}$. Several additional sources have since been identified from the LRS database (Hrivnak \& Kwok 1991) or from ground-based spectroscopy using the UKIRT CGS3 spectrometer (Kwok, Hrivnak, \& Geballe 1995; Justtanont et al. 1996). This bring the number of $21 \mu \mathrm{m}$ sources to 11 .

The feature continues to be found only in PPNe (but see Henning, Chan, \& Assendorp 1996, who recently claim to find it in several YSOs), and only in those which are clearly C-rich. The fact that it is seen only in PPNe indicates that it has a transitory or frail nature. It is not seen in the AGB stars, where it is either not present or present but not excited. In the $\mathrm{PNe}$, it is not seen, presumably because it is destroyed by the uv radiation field.

In Table 1 , we list the $21 \mu \mathrm{m}$ sources and some of the particular properties common to most of them. The strong correlation with carbon and $\mathrm{PAH}$ features is evident, and most also have strong HCN measurements. In fact, on this basis, we predict two new $21 \mu \mathrm{m}$ sources, which we plan to confirm with ISO. Suggestions continue to be made for the identification of the $21 \mu \mathrm{m}$ feature (see Justtanont et al. 1996).

$30 \mu \mathrm{m}$ : A very broad feature at $30 \mu \mathrm{m}$ is known from $\mathrm{KAO}$ observations to exist in the spectra of a range of C-rich evolved stars, from AGB stars to $\mathrm{PNe}$ (Cox 1993). This feature has been seen in the spectra of at least 5 PPNe, including the Egg nebula (Omont et al. 1995). This is a strong fea- 


\section{B. Hrivnak}

TABLE 1. List of $21 \mu \mathrm{m}$ sources and their common properties

\begin{tabular}{ccclll}
\hline ID & $21 \mu \mathrm{m}$ & $30 \mu \mathrm{m}$ & $\mathrm{SpT}$ & Optical & IR features (PAH) \\
\hline & & & & & \\
$04296+3429$ & strong & $\ldots$ & $\mathrm{G} 0 \mathrm{Ia}$ & $\mathrm{C}_{2}, \mathrm{C}_{3}, \mathrm{CN}$ & $3.3,3.4-3.5,7.7,11.3$ \\
$05113+1347$ & medium & $\ldots$ & $\mathrm{G} 7 \mathrm{Ia}$ & $\mathrm{C}_{2}, \mathrm{C}_{3}, \mathrm{CN}$ & $3.3,11.3$ \\
$05341+0852$ & weak & $\ldots$ & F6 I & $\mathrm{C}_{2}, \mathrm{C}_{3}, \mathrm{CN}$ & $3.3,3.4-3.5,7.7,11.3$ \\
$07134+1005$ & v. strong & strong & F5 I & $\mathrm{C}_{2}, \mathrm{CN}$ & $3.3,6.9$ \\
$19500-1709$ & v. weak & $\ldots$ & F3 I & $\ldots$ & $\ldots$ \\
$20000+3239$ & weak & v. strong & G7 Ia & $\mathrm{C}_{2}, \mathrm{CN}$ & $7.7,11.3$ \\
AFGL 2688 & weak & medium & F5 Iae & $\mathrm{C}_{2}, \mathrm{C}_{3}, \mathrm{CN}$ & $3.3,3.4-3.5$ \\
$22223+4327$ & medium & $\ldots$ & G0 Ia & $\mathrm{C}_{2}, \mathrm{C}_{3}, \mathrm{CN}$ & $\ldots$ \\
$22272+5435$ & strong & v. strong & G5 Ia & $\mathrm{C}_{2}, \mathrm{C}_{3}, \mathrm{CN}$ & $3.3,3.4-3.5,6.9,7.7,11.3$ \\
$22574+6609$ & medium & $\ldots$ & $\ldots$ & $\ldots$ & $7.7,11.3$ \\
$23304+6147$ & strong & v. strong & G2 Ia & $\mathrm{C}_{3}, \mathrm{CN}$ & $7.7,11.3$ \\
"Predicted New Sources” & & & & \\
$02229+6208$ & not obs. & $\ldots$ & late-G I & $\mathrm{C}_{2}, \mathrm{C}_{3}$ & $\ldots$ \\
$07430+1115$ & not obs. & $\ldots$ & mid-G I & $\mathrm{C}_{2}, \mathrm{C}_{3}$ & $3.3,3.4,11.3$ \\
\hline
\end{tabular}

References: (1) Kwok, Volk, \& Hrivnak 1989; (2) Hrivnak \& Kwok 1991; (3) Buss et al. 1993; (4) Justtanont 1996; (5) Omont et al. 1995; (6) Hrivnak 1995; (7) Geballe et al. 1992; (8) Buss et al. 1990.

ture in some PPNe; in IRAS $22272+5435$ it makes up $20 \%$ of the bolometric luminosity. All 5 of these PPNe also possess the $21 \mu \mathrm{m}$ feature; however, the strength of the two features is not correlated. The best identification thus far for the origin of this $30 \mu \mathrm{m}$ feature is $\mathrm{MgS}$ (Goebel 1980; Goebel \& Moseley 1985), or a mixture of $\mathrm{MgS}$ and amorphous carbon grains (Omont et al. 1995), or a mixture of MgS and FeS (10\%) (Szczerba et al. 1996).

We can eagerly look forward to the good ISO data to extend our knowledge of the prevalence of the 21 and $30 \mu \mathrm{m}$ features, and to allow us to investigate substructure in these features and other new features. This will be exciting, and undoubtedly there will be much to learn here.

\section{Circumstellar Envelopes: Morphology}

\subsection{INTRODUCTION}

The ability to resolve images of PPNe can allow one to study the processes that go into shaping the PN nebulae. While the CSEs of AGB stars are generally spherically symmetric, although perhaps clumpy, the nebulae of most PNe display axial (not spherical) symmetry. This PN morphology is thought to arise from the interaction between the fast wind of the central star and the remnant CSE of the AGB progenitor. The axial symmetry thus requires a density enhancement in the plane perpendicular to the axis 


\section{PROTO-PLANETARY NEBULAE}

(Balick 1987). Detailed hydrodynamic models confirm that this will produce the appearance of the observe PNe (Frank \& Mellema 1994; Mellema \& Frank 1995). The cause of this density enhancement has been attributed to (a) the gravitational effect of a binary companion, (b) rotation, perhaps amplified by a binary companion, or (c) magnetic fields. These mechanisms have been discussed at this conference (see Livio). This axial symmetry must arise or be enhanced during the PPN evolution. Thus the study of the morphology of PPNe holds the potential to allow us to see how this asymmetry develops.

The fact that the first good candidates for PPNe discovered - AFGL 2688 (Egg nebula) and AFGL 618 - both show a bipolar morphology encourages us that the imaging of PPNe will be fruitful. Direct imaging of additional PPNe has been obtained over the last several years, as discussed below. A polarization study by Trammell, Dinerstein, \& Goodrich (1994) obtains results that are, in general, supportive of the results of these imaging studies.

\subsection{MOLECULAR-LINE CO}

Large objects can be resolved and mapped in $\mathrm{CO}$, and this has been done for IRAS 19114+0002 (Bujarrabal, Alcolea, \& Planesas 1992) and 22272+5435 (Dayal et al., this conference). Recent results from the Nobeyama Millimeter Array have been presented by Deguchi (this conference). New millimeter and sub-millimeter arrays will give the ability to resolve the CSEs of many $\mathrm{PPNe}$ and to study the physical conditions.

\subsection{MID-INFRARED IMAGING}

Mid-infrared imaging can allow one to see the dust envelope directly, particularly the inner edge. Recent advances in mid-infrared array technology are being exploited by several groups to study PPNe at 10 and $20 \mu \mathrm{m}$. Based upon published studies and preprints, the results of these studies are as follows: (a) two O-rich PPNe have been imaged and resolved, one of which shows a torus (HD 161796, Skinner et al. 1994) and one of which is round (IRAS 19114+0002, Hawkins et al. 1995); (b) four C-rich, $21 \mu \mathrm{m}$ sources have been imaged and three resolved: one shows a torus and two are elliptical (Meixner et al. 1996). Additional results are presented by Dayal et al., Kömpe, and Van de Steene \& van Hoof (this conference), and a summary is given by Meixner in this conference. In addition, a detailed imaging study of the Egg nebula at near-infrared and mid-infrared has been made by Skinner et al. (1996). 


\section{B. Hrivnak}

\subsection{VISIBLE \& NEAR-INFRARED IMAGING}

Two new bipolar PPNe have recently been discovered, IRAS 17150-3224 and 17441-2411 ( $\mathrm{Hu}$ et al. 1993; Kwok et al. 1996). In a recently completed study at a resolution of $0.7^{\prime \prime}$ on the Canada-France-Hawaii Telescope, 10 additional, smaller PPNe have been found to be extended, with a typical size of $4^{\prime \prime}$ (Hrivnak et al. 1996). The shapes are as follows: elliptical - 6 (including 1 in which the PA varied), round - 3, and 1 with elliptical outer and round inner intensity contours.

Of course new observations have been made at even higher resolution in visible light with the HST. Some of these images, such as the Egg nebula, are familiar because of their wide distribution, while others, such as the Red Rectangle, were shown in this conference (Bond). The high-resolution images of the Egg nebula resolve the bipolar lobes into two pairs of radial "searchlight beams" and a series of approximately concentric arcs, suggesting reflection from dust emitted with a spacing of about 400 years between arcs. These high-resolution studies will help to determine if mass loss is steady or episodic.

These observations make it clear that non-spherical, axially-symmetric morphology is common in PPNe, and that it is developed by early in the transition stage from the AGB to the PN.

\section{Star: Chemistry}

There have been previous abundance studies of several stars classified as high-latitude $\mathrm{F}$ supergiants. These objects are now generally regarded as low-mass post-AGB and differ from those which we are discussing in that they are mostly without massive CSEs (little mid-infrared excess), and probably will not evolve into $\mathrm{PNe}$. The results of the abundance studies of these objects are not as clear as one might have hoped for (see Luck 1993; Van Winckel 1995); while they appear to be metal-poor, the expected carbon and s-process enhancements from the dredge-up of the results of $\mathrm{He}$ burning are not seen. New studies have shown some of these to be binaries, and some of their peculiar chemical abundance patterns have received a nice explanation as chemical fractionation within the context of a binary star model (Waters, this conference).

In this review, we have restricted our discussion to those which we call $\mathrm{PPNe}$ (strong mid-infrared excess). For these, several recent abundance studies have been published. These tend to clearly support the post-AGB nature of these objects, and most of them display abundance patterns indicating the products of a third dredge-up. This is seen in particular in the enhancement of carbon and s-process elements, and in some cases in a low metal abundance indicating a Pop II or thick disk population, and 
thus old stars. These results are collected in Table 2. They agree with the expectation of post-AGB stars evolving to become PNe.

TABLE 2. Abundance studies of central stars of PPNe

\begin{tabular}{|c|c|c|c|c|c|c|c|c|}
\hline ID & $\mathrm{SpT}$ & $\begin{array}{c}\log g \\
(\mathrm{cgs})\end{array}$ & {$[\mathrm{Fe} / \mathrm{H}]$} & $\mathrm{C} / \mathrm{O}$ & {$[\mathrm{C} / \mathrm{Fe}]$} & {$[\mathrm{s} / \mathrm{Fe}]$} & Chemistry & Ref. \\
\hline $05341+0852$ & F6 I & 0.5 & -1.0 & 2 & +1.0 & +2.2 & $\mathrm{C}$ & (5) \\
\hline \multirow[t]{2}{*}{$07134+1005$} & F5 I & 0.1 & -1.0 & 3 & +1.1 & +2.0 & $\mathrm{C}$ & (3) \\
\hline & & 0.5 & $\leq-1.0$ & $\sim 1$ & +1.0 & & & (6) \\
\hline HD 161796 & F3 Ib & 0.3 & $-\overline{-0.3}$ & 0.8 & +0.3 & 0.0 & $\mathrm{O}$ & (7) \\
\hline $18095+2704$ & F3 Ib & 1.0 & -0.8 & 0.7 & $\sim+0.5$ & -0.1 & $\mathrm{O}$ & (3) \\
\hline $19114+0002$ & F5 Ia ${ }^{*}$ & 1.3 & -0.1 & $\ldots$ & $\ldots$ & $\sim+0.7$ & $\mathrm{O}$ & (4) \\
\hline $19500-1709$ & A2-3 I ${ }^{*}$ & 1.0 & -0.5 & $\sim 1$ & +0.9 & +1.2 & $\mathrm{C}$ & (2) \\
\hline $22272+5435$ & G2 Ia & 0.5 & -0.5 & $\sim 12$ & +1.7 & +2.5 & $\mathrm{C}$ & (1) \\
\hline
\end{tabular}

* Spectral types assigned on basis of model atmosphere fitting.

References: (1) Zăs, Klochkova, \& Panchuk 1995; (2) Van Winckel, Waelkens, \& Waters 1996; (3) Klochkova 1995; (4) Zăs et al. 1996; (5) Reddy et al. 1996; (6) Parthasarathy, Garcia Lario, \& Pottasch 1992; (7) Luck, Bond, \& Lambert 1990.

\section{Star: Variability}

Variability in the central stars of PPNe can arise from either pulsations or a binary companion. AGB stars, of course, pulsate with long periods, and there are classes of post-AGB which are known to pulsate - RV Tau $(\mathrm{P}=50-150 \mathrm{~d}$, alternating deep and shallow minima, $\mathrm{SpT}=\mathrm{F}-\mathrm{K})$ and $\mathrm{UU}$ Her $(\mathrm{P}=40-100 \mathrm{~d}$, small amplitude, SpT $=\mathrm{F})$. Variability, particularly in radial velocity, can arise from a binary companion, and such a companion can be very important in determining an axis of symmetry for the AGB mass loss and the subsequent shaping of the PN.

These reasons motivated us to undertake a high-resolution radial velocity study of the brighter PPN candidates. Nine bright PPNe were observed for radial velocity variations over 5 seasons; all 9 were found to vary, with an average velocity range of $10 \mathrm{~km} \mathrm{~s}^{-1}$. For 3 of these, a consistent period was found: $18095+2704(\mathrm{~F} 3 \mathrm{Ib}, \mathrm{P}=109 \mathrm{~d}), 22223+4327$ (G0 Ia, P = $89 \mathrm{~d})$, $22272+5435$ (G5 Ia, P = $127 \mathrm{~d}$ ).

To better understand the nature of these objects, and to extend the study to fainter PPN candidates, a photometric study was initiated in 1994. We are presently monitoring $\sim 40 \mathrm{PPN}$ candidates for photometric variability. Some interesting initial results have been found. (1) Almost all of the 40 show low-amplitude light variations, with typical variations of $0.15-0.35$ 


\section{B. Hrivnak}

mag in V. (2) Periodic light variation has been found in 9 objects thus far, with periods ranging from $25-146 \mathrm{~d}$. These objects all have spectral types F-G. (3) The 3 with periodic velocity variations also display light variations with the same period, but both the light and velocity curves show changes in the amplitude of the variations. (4) Short timescale variations $(<10 \mathrm{~d})$ have been found in several of the objects, particularly those of early spectral types, $\mathrm{O}-\mathrm{B}$. (5) There appears to be a general trend of shorter $\mathrm{P}$ with earlier spectral type. The nature of these variations points to pulsations, rather than a binary nature. More details of this study are presented at this conference (Hrivnak \& Lu; see also Hrivnak \& Lu 1996).

\section{Theory \& Modeling}

Evolutionary models of PPNe must include both the evolution of the star and any ongoing mass loss from the atmosphere. Following the earlier models of Schönberner (1983) are more recent models by Blöcker (1995), Schönberner, Blöcker, \& Marten (1995), and Schönberner (this conference) and also models by Vassiliadis \& Wood (1994) and Wood (this conference). These evolutionary models derive transitional ages of a few thousand years between the end of the AGB mass loss and the beginning of the PN stage.

Modelling of the SED has been carried out by a few groups, to yield the parameters of the circumstellar dust shells. Most models have assumed spherical symmetry, and have fitted parameters such as the inner dust shell radius and density distribution in the shell, assuming a certain grain size and opacity and a stellar temperature and luminosity (Volk \& Kwok 1988, 1989; Gürtler, Kömpe, \& Henning 1996). Szczerba et al. (1996) recently modelled the SED of IRAS $22272+5435$ using PAH and amorphous carbon grains of different sizes and an empirical opacity function to fit the 21 and $30 \mu \mathrm{m}$ features. With the availability of resolved images, which show some nebulae to not be spherically symmetric, a second constraint is added, the morphology. Recently Skinner et al. (1996) fitted an axially-symmetric model to the SED and the bipolar morphology of the Egg nebula. Evolutionary models of the SEDs of PPNe have been carried out by Volk (1992) and more recently by Steffan et al. (this conference), assuming spherical symmetry.

\section{Conclusions \& A Look To The Future}

We will list a few brief conclusions drawn from this review of PPNe. We have recently found that almost all of them appear to pulsate. Abundance patterns are observed to fit with those expected for post-AGB stars which have experience a third dredge up. Non-spherical (bipolar or elliptical) morphologies are clearly evident in PPNe, even in the cooler (younger) ones. 


\section{PROTO-PLANETARY NEBULAE}

These studies will be extended by the new images obtained by HST with WFPC2 and NICMOS, with adaptive optics, and with diffraction-limited observations in the near-infrared and mid-infrared on the new class of $8 \mathrm{~m}$ and $10 \mathrm{~m}$ telescopes. As we look to the future, and in particular the next $\mathrm{PN}$ conference, we also eagerly look forward to the results from ISO to learn more about the chemical and physical properties of the CSEs of PPNe.

\section{Acknowledgments}

I wish to acknowledge with gratitude my close colleague in much of this research on PPNe, Sun Kwok, who unfortunately was not able to attend this conference. Thanks also to Kevin Volk, Phil Langill, and Wen Lu for their collaboration in parts of this research. Support was provided by NSF (AST-9018032, AST-9315107) and NASA (NAG5-1223, NAG8-232).

\section{References}

Bakker, E.J., Waters, L.B.F.M., Lamers, H.J.G.L.M., Trams, N.R., \& Van der Wolf, F.L.A. 1996, $A \& A, 310,893$

Bakker, E.J., van Dishoeck, E.F., Waters, L.B.F.M., \& Schoenmaker, T. 1997, $A \& A$, in press

Balick, B. 1987, $A J, 94,671$

Blöcker, T. 1995, A\& A, 299, 755

Bujarrabal, V., Alcolea, J., \& Planesas, P. 1992, A\&A, 257, 701

Buss, Jr., R.H., et al. 1990, ApJ, 365, L23

Buss, Jr., R.H., et al. 1993, $A p J, 415,250$

Cox, P. 1993, in Astronomical Infrared Spectroscopy, ed. S. Kwok, ASP Conf. Series, 41, 163

Frank, A., \& Mellema, G. 1994, ApJ, 430, 800

Geballe, T.R., Tielens, A.G.G.M., Kwok, S., \& Hrivnak, B.J. 1992, ApJ, 387, L89

Goebel, J.H. 1980, BAAS, 14, 858

Goebel, J.H., \& Moseley, S.H. 1985, ApJ, 290, L35

Gürtler, J., Kömpe, C., \& Henning, Th. 1996, $A \& A, 305,878$

Hawkins, G.W., et al. 1995, ApJ, 452, 314

Henning, Th., Chan, S.J., \& Assendorp, R. 1996, $A \& A, 312,511$

Hrivnak, B.J. 1995, $A p J, 438,341$

Hrivnak, B.J., \& Kwok, S. 1991, ApJ, 368, 564

Hrivnak, B.J., Kwok, S., \& Geballe, T.R. 1994, $A p J, 420,783$

Hrivnak, B.J., Kwok, S., \& Volk, K.M. 1989, ApJ, 346, 265

Hrivnak, B.J., \& Lu, W. 1996, in IAU Symp. 177: The Carbon Star Phenomenon, ed. R.F. Wing, (Dordrecht: Kluwer), in press

Hrivnak, B.J., et al. 1996, in preparation

Hu, J.Y., Slijkhuis, S., Nguyen-Q-Rieu, \& de Jong, T. 1993, A\& A, 273, 185

Justtanont, K., Barlow, M., Skinner, C.J., Roche, P.F., Aitken, D.K., \& Smith, C.H. 1996, $A \& A, 309,612$

Klochkova, V.G. 1995, MNRAS, 272, 710

Kwok, S. 1993a, in IAU Symp. 155: Planetary Nebulae, ed. R. Weinberger, A. Acker (Dordrecht: Kluwer), 263

Kwok, S. 1993b, Ann. Rev. A\&A, 31, 63

Kwok, S., Hrivnak, B.J., \& Geballe, T.R. 1995, ApJ, 454, 394 


\section{B. Hrivnak}

Kwok, S., Hrivnak, B.J., Zhang, C.Y., \& Langill, P.P. 1996, ApJ, 472, 287

Kwok, S., Volk, K.M., \& Hrivnak, B.J. 1989, ApJ, 345, L51

Luck, R.E. 1993, in Luminous High Latitude Stars, ed. D.D. Sasselov, ASP Conf. Series, 45,87

Luck, R.E., Bond, H.E., \& Lambert, D.L. 1990, $A p J, 357,188$

Meixner, M., et al. 1996, preprint

Mellema, G., \& Frank, A. 1995, MNRAS, 273, 401

Omont, A. Loup, C., Forveille, T., te Lintel Hekkert, P., Habing, H., \& Sivagnanam, P. 1993, $A \& A, 267,515$

Omont, A., et al. 1995, $A p J, 454,819$

Oudmaijer, R.D, Waters, L.B.F.M., van der Veen, W.E.C.J., \& Geballe, T.R. 1995, A\&A, 299,69

Parthasarathy, M., Garci Lario, P., \& Pottasch, S.R. 1992, A\&A, 264, 159

Parthasarathy, M., \& Pottasch, S.R. 1986, A\&A, 154, L16

Reddy, B.E., \& Parthasarathy, M. 1996, preprint

Reddy, B.E., Parthasarathy, M., Gonzalez, G., \& Bakker, E.J. 1996, preprint

Schönberner, D. 1983, ApJ, 272, 708

Schönberner, D., Blöcker, T., \& Marten, H. 1995, in Asymmetrical Planetary Nebulae, ed. A. Harpaz, N. Soker, Ann. Israel Phys. Soc., 11, 81

Skinner, C.J., et al. 1994, $A p J, 423$, L135

Skinner, C.J., et al. 1996, preprint

Szczerva, R. et al. 1996, preprint

Trammell, S.R., Dinerstein, H.L. \& Goodrich, R.W. 1994, AJ, 108, 984

Van Winckel, H. 1995, PhD thesis, Catholic University of Leuven

Van Winckel, H., Waelkens, C., \& Waters, L.B.F.M. 1996, A\&A, 306, L37

Vassiliadis, E., \& Wood, P. R., 1994, ApJS, 92, 125

Volk K.M. 1992, ApJS, 80, 347

Volk K.M., \& Kwok S. 1988, ApJ, 331, 435

Volk K.M., \& Kwok S. 1989, ApJ, 342, 345

Zăs, L., Klochkova, V.G., \& Panchuk, V.E. 1995, MNRAS, 275, 764

Zăs, L., Klochkova, V.G., Panchuk, \& Spēlmanis, R. 1996, preprint 